\title{
EULER ELASTICAE IN THE PLANE AND THE WHITNEY-GRAUSTEIN THEOREM
}

\author{
S. AVVAKUMOV, O. KARPENKOV, A. SOSSINSKY
}

\begin{abstract}
In this paper, we apply classical energy principles to Euler elasticae, i.e., closed $\mathcal{C}^{2}$ curves in the plane supplied with the Euler functional $U$ (the integral of the square of the curvature along the curve). We study the critical points of $U$, find the shapes of the curves corresponding to these critical points and show which of the critical points are stable equilibrium points of the energy given by $U$, and which are unstable. It turns out that the set of stable equilibrium points coincides with the set of minima of $U$, so that the corresponding shapes of the curves obtained may be regarded as normal forms of Euler elasticae. In this way, we find the solution of the Euler problem (set in 1744) for plane closed elasticae. As a by-product, we obtain a "mechanical" proof of the Whitney-Graustein theorem on the classification of regular curves in the plane up to regular homotopy (in the particular case of $\mathcal{C}^{2}$ curves). Besides mathematical theorems, our work includes a computer graphics software which shows, as an animation, how any plane curve evolves to its normal form under a discretized version of gradient descent along the (discretized) Euler functional.
\end{abstract}

\section{INTRODUCTION}

The aim of this paper is to test how the energy functional approach works on the moduli space of all regular $\mathcal{C}^{2}$ curves in the plane $\mathbb{R}^{2}$ in the case of the Euler functional

$$
U(\gamma)=\int_{0}^{2 \pi}(\kappa(\gamma(s)))^{2} d s
$$

where $\gamma: \mathbb{S}^{1} \rightarrow \mathbb{R}^{2}$ is a curve of length $2 \pi, s$ is the arclength parameter, and $\kappa(\gamma(s)$ is the curvature of $\gamma$ at the point $s$. Thus we view regular homotopy classes of regular plane curves through the prism of the Euler functional.

Let us start with a general remark. Suppose that one has a configuration space $S$ that is split in several connected components and the main task is to check if two elements of $S$ are in the same connected component.

Then there are two main strategies to do this: combinatorial and mechanical. The combinatorial strategy is based on finding a (hopefully complete) invariant distinguishing connected components. The mechanical strategy is as follows: one finds an appropriate functional (which is sometimes called energy); then gradient or descent flow on $S$ is performed along the functional

Date: 7 November 2012.

Key words and phrases. curvature minimization, Euler functional, Whitney-Graustein theorem. 
and the problem is reduced to comparing local minima of this flow (these minima are called normal forms). For smooth enough moduli spaces $S$ and an appropriate choice of energy functionals, the gradient flows corresponding to these energies take almost all (except for a measure zero set) configurations of $S$ to the corresponding local minima.

A good example where both strategies are applied is the theory of knots and links. The combinatorial approach is broadly studied in classical knot theory, where many beautiful invariants have been devised (Alexander and Jones polynomials, Vassiliev invariants, Khovanov homology, etc.). The mechanical strategy, i.e., the idea of defining energy functionals for knots is due to H. K. Moffat [13]. It was further developed by W. Fukuhara [6], J. O'Hara in [14], [15], [16], [17], M. H. Freedman with various co-authors in [4], [5] [2], by D. Kim, R. Kusner in [11], O. Karpenkov in [8], [9], etc. Some aspects of the intermediate step between classical and mechanical approaches is discussed in [7]. In practice, the energy techniques work well, but the mathematical justification of theoretical questions appears too complicated to be resolved at the present time. For instance, it is not proven that the unknot has a unique local minimum with respect to the famous Möbius functional invented by O'Hara.

In this paper, rather than applying the energy techniques to knots and links, we test the mechanical approach on regular curves in the plane, which are simpler objects than knots (to which we intend to return in subsequent publications). The combinatorial approach to the study of regular curves in the plane yields the classical Whitney-Graustein theorem, which provides a classification of curves up to regular homotopy by means of a simple complete invariant - the Whitney index or winding number, which is the number of revolutions effected by the tangent vector to the curve at a mobile point when the mobile point goes once around the curve. Here we discuss the mechanical approach to the study of regular plane curves of class $\mathcal{C}^{2}$ using the Euler functional $U$ (the integral along the curve of the square of the curvature) and describe the corresponding normal forms with respect to $U$. It turns out that each regular homotopy class has a unique normal form (Theorem 2.2) and the normal form is a complete invariant of regular homotopy classes. In the case of a nonzero Whitney index, the normal form is a circle passed once or several times, otherwise it is Bernoulli's closed $\infty$-shaped elastica (the figure eight curve). These normal forms are obtained by gradient descent along values of $U$ in the space of curves. Computer animations of the discretization of this process (created by the first-named author) are available at [1].

This paper is organized as follows. We start in Section 1 with the necessary definitions and preliminaries. In Section 2, we formulate the main results of this paper (Theorems 1.3 and 2.2) on normal forms of regular curves. In Section 3, we describe the algorithm on which the animations are based. Finally, in Section 4, we give technical details and proofs.

In more detail, Section 4, in which critical points of the Euler functional $U$ are studied, consists of five subsections. In Subsection 4.1, we discuss the relationship of critical points to the simple pendulum. We study differentiability questions of critical curves in Subsection 4.2. In Subsection 4.3, we prove that all critical curves are either circles passed once or several times or Bernoulli's closed elastica passed once or several times. We construct a deformation of critical Bernoulli's closed elastica (passed more than once) reducing the value of Euler's functional in Subsection 4.4. Finally, we conclude the proofs of Theorem 1.3 and Theorem 2.2 in Subsection 4.5. 


\section{Preliminaries}

1.1. Gauss representation of regular curves. A plane closed curve $\gamma: \mathbb{S}^{1} \rightarrow \mathbb{R}^{2}$ is regular if it possesses a nonzero derivative (tangent vector) at each point $\gamma(t)$.

For a regular curve $\gamma$, we consider a function $\alpha:[0,2 \pi] \rightarrow \mathbb{R}$ such that

$$
\dot{\gamma}(t)=(\cos \alpha(t), \sin \alpha(t)) \text {. }
$$

We then say that $\alpha$ is a Gauss representation of $\gamma$. (Here and later by $\dot{g}$ we denote the derivative $\partial g / \partial t$.) Notice that a pair of curves having the same Gauss representations coincide after a translation by some vector.

In the Gauss representation, we have $\kappa=\dot{\alpha}$ and hence the Euler functional is as follows:

$$
U(\alpha)=\int_{0}^{2 \pi} \dot{\alpha}^{2} d t .
$$

Proposition 1.1. A continuous function $\alpha$ is a Gauss representation of some regular curve if the following conditions hold:

$$
\begin{aligned}
\int_{0}^{2 \pi} \cos \alpha(t) d t & =\int_{0}^{2 \pi} \sin \alpha(t) d t=0 ; \\
\alpha(0) & =\alpha(2 \pi) .
\end{aligned}
$$

The proof is obvious.

1.2. Normal forms of regular curves. The functional

$$
\int_{0}^{2 \pi} \kappa^{2}(\gamma(t)) d t,
$$

defined for any plane closed curve of class $\mathcal{C}^{2}$ is called the Euler functional; we denote it by $U$. (Notice that in our previous paper [7] we considered a wider class of functionals. The Euler functional was denoted there by $U_{x^{2}}$ ). A curve supplied with the Euler functional is traditionally (see e.g. [10] called an Euler elastica.

Definition 1.2. The normal form of a regular plane $\mathcal{C}^{2}$ curve with respect to the Euler functional is a plane curve for which the value of $U$ is a local minimum.

Notice that unstable equilibrium points for $U$ do not not give normal forms. Gradient descent reaches such points with zero probability (such an event practically never occurs in real life).

The main result of the present paper is the following theorem.

Theorem 1.3. (i) Any critical regular curve of the Euler functional is either a circle passed several times or Bernoulli's $\infty$-shaped closed elastica passed several times:
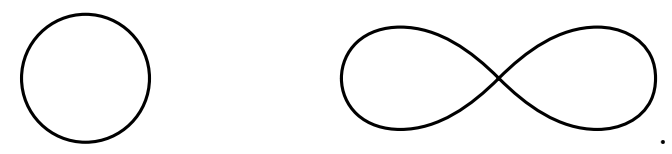

(ii) A Bernoulli closed $\infty$-shaped elastica passed several times is not stable. 
(iii) A circle passed once or several times and the Bernoulli closed elastica passed once are local minima.

This result gives a complete solution of the Euler problem for closed elasticas in the plane. Note that the same result was obtained independently by Yu.Sachkov (see [19]) by a different, much more laborious method (involving the Pontryagin maximum principle).

\section{The Whitney-Graustein theorem via Euler Elasticas}

Definition 2.1. The Whitney index of a regular plane curve $\gamma$ is the degree of the corresponding Gauss map, taken with a sign (i.e., the number of clockwise rotations effected by the tangent vector when its origin travels once around the curve). We denote it by $\omega(\gamma)$.

For a Gauss representation $\alpha$ of $\gamma$, the following obviously holds:

$$
\omega(\gamma)=\int_{\gamma} \alpha(t) d t .
$$

We recall the classical statement of the Whitney-Graustein theorem: Regular plane curves are classified up to regular homotopy by their Whitney index, i.e., two regular plane curves are regularly homotopic if and only if they have the same Whitney index.

The following statement immediately follows from Theorem 1.3 below and the fact that the normal form curves appearing in Theorem 1.3 have Whitney indices $0, \pm 1, \pm 2, \ldots, \pm n, \ldots$ respectively.

Theorem 2.2. (On Euler normal forms.) Two regular plane curves of class $\mathcal{C}^{2}$ are regularly homotopic if and only if they have the same normal form with respect to the Euler functional $U$.

Remark 2.3. It is not hard to construct different descent flows, we omit the related analysis. The software [1] described in the next section gives a practical, fast (a few seconds for reasonably simple curves), and very visual method for determining the regular homotopy type of a plane curve: one simply observes the evolution of the curve until it reaches its normal form, which determines the regular homotopy type. In general it would be interesting to have a constructive proof that such flows always end up in a smooth critical realization. The second question which we do not touch here is the rigorous construction of the gradient flow.

\section{Normal Forms VIA COMPUTER ANimations}

In this section we describe our computer software, which shows how a given curve evolves to its normal form, and describe the underlying algorithm.

The software may be downloaded from [1]. It is a user friendly interactive animation and can be viewed on any PC running with Windows XP (or any later version). Once the program (exe file) is downloaded and activated, the user simply draws the required curve on the screen with the mouse (or by means of the touch pad). The curve begins to evolve, progressively changing its shape until it arrives to its normal form - a circle of radius $\rho, \rho / 2, \rho / 3, \ldots$, or a Bernoulli $\infty$-shaped curve of length $2 \pi \rho$. The evolution of the curve in the animation to its normal form takes a minute or a few minutes for moderately complicated curves. 
The descent algorithm, which is an iterative process, can be briefly described as follows. First, the computer transforms the input curve into a closed polygonal line (usually with selfintersections) with edges $e_{i}$ of the same (tiny) length and vertices $v_{i}$, where $e_{i}=\left[v_{i-1}, v_{i}\right]$, $i=1, \ldots N$; here $N$ is a parameter of our program.

The discrete version of our functional is

$$
\widehat{U}:=\sum_{i} \tan ^{2}\left(\alpha_{i} / 2\right)
$$

where $\alpha_{i}$ is the angle between the continuation of the edge $e_{i}$ and the edge $e_{i+1}$.

At each step of the algorithm, for each vertex $v_{i}$, two "forces" (vectors) $s_{i}$ and $r_{i}$ are computed. The vector $s_{i}$ (the straightening out force) is calculated according to the formula

$$
s_{i}=-C_{1}\left(\frac{\partial}{\partial x_{i}}+\frac{\partial}{\partial y_{i}}\right)(\widehat{U}),
$$

where $v_{i}=\left(x_{i}, y_{i}\right)$ and $C_{1}$ is a positive constant; here the partial derivatives $\partial \widehat{U} / \partial x_{i}$ and $\partial \widehat{U} / \partial y_{i}$ are calculated approximately as finite differences.

The vector $r_{i}$ (the resilience force) is calculated according to the formula

$$
r_{i}:=C_{2}\left(v_{i+1}-v_{i}\right)\left(\left|v_{i+1}-v_{i}\right|-d_{i}\right)+C_{2}\left(v_{i-1}-v_{i}\right)\left(\left|v_{i}-v_{i-1}\right|-d_{i-1}\right),
$$

where $C_{2}$ is a positive constant, $|v|$ is the Euclidean norm of the vector $v$, and $d_{i}$ is the Euclidean distance between the vertices $v_{i}$ and $v_{i+1}$ at the initial moment.

Then each of the points $v_{i}$ is shifted by the vector $s_{i}+r_{i}$, a new polygonal line is obtained, and the algorithm goes on to the next step.

The constants $C_{1}$ and $C_{2}$ are parameters of our program and are chosen so that the lengths of the edges, as well as the total length of the curve, do not change significantly during the descent process.

The iteration process continues endlessly (there is no termination command in the program). However, after a short interval of time, not more than a few minutes in all our experiments (performed with $N=100$ ), the modifications in the shape of the curve become invisible to the naked eye. We then consider the iteration process as terminated and regard the shape of the obtained curve as the normal form of the input curve. In all our experiments, the obtained curve was always one of the normal forms predicted by Theorem 1.3.

Note that this result is not a mathematical theorem, but an experimental fact. We intend to return to its mathematical justification in subsequent publications.

\section{Critical points of the Euler functional}

In this section, we study critical points of the Euler functional $U$ and prove our main result (Theorem 1.3) and from it derive the proof of Theorem 2.2.

First, we study the case of twice differentiable Gauss representations, for which the question is reduced to the equation of the simple pendulum. Secondly, we show that all critical values of the Euler functional indeed possess twice differentiable Gauss representations. The most complicated case is the case of zero Whitney index: the critical points are the Bernoulli's closed $\infty$-shaped curves passed several times. We explicitly construct deformations of these closed elasticae passed several times that reduce the energy (which proves that these critical curves are not local minima). In addition, we prove that there are no simple closed curves satisfying the equation of the simple pendulum other than circles. 
4.1. The first variation of Euler functional at a critical elastica. Let $\gamma$ be a smooth enough regular plane curve with Gauss representation $\alpha$.

Consider a variation $\alpha+h \beta$ with a small parameter $h$. First, since we vary a closed curve, the variation $\alpha+h \beta$ infinitesimally satisfies conditions (1) above, i.e., the derivative $\frac{d}{d h}$ of the corresponding integrals equals zero, which is equivalent to

$$
\begin{gathered}
\int_{0}^{2 \pi} \sin (\alpha(t)) \beta(t) d t=\int_{0}^{2 \pi} \cos (\alpha(t)) \beta(t) d t=0, \\
\beta(0)=\beta(2 \pi) .
\end{gathered}
$$

Secondly, for the case in which $\alpha$ is a critical elastica, the first variation is zero. Hence

$$
\int_{0}^{2 \pi} \dot{\alpha} \dot{\beta} d t=0,
$$

which is equivalent to

$$
\int_{0}^{2 \pi} \alpha \ddot{\beta} d t=0 .
$$

Now we have an equation for critical points even if the Gauss representation is only continuous.

There is another equivalent representation of the equation for the first variation

$$
\int_{0}^{2 \pi} \ddot{\alpha} \beta d t=0 .
$$

The last equation holds for any variation $\beta$ satisfying Equations (2), therefore, we have the following statement.

Corollary 4.1. Let $\gamma$ have a twice differentiable Gauss representation $\alpha$. Suppose that $\gamma$ is critical for the Euler functional. Then there exist constants $C_{1}$ and $C_{2}$ such that $\alpha$ satisfies

$$
\ddot{\alpha}=C_{1} \cos \alpha+C_{2} \sin \alpha .
$$

Remark 4.2. If $C_{1}=C_{2}=0$ then we have the equation of circles: $\ddot{\alpha}=0$. If at least one of the constants $C_{1}$ and $C_{2}$ is not equal to zero, then after an appropriate Euclidean transformation, we obtain the equation for the simple pendulum

$$
\ddot{\alpha}+\omega^{2} \sin \alpha=0
$$

for some nonnegative constant $\omega$.

4.2. Smoothness of critical elasticae. Let us prove the smoothness of critical elasticae. The proof of this assertion is traditionally missing in the literature, it is usually supposed that the curve is smooth enough.

Proposition 4.3. The Gauss representation of a critical elastica is twice differentiable at any point.

We prove the assertion of the proposition in three steps. First, we show that the Gauss representation is continuous. Secondly, we show that it is continuously differentiable. Finally, we prove that it is twice differentiable. 


\subsubsection{Continuity of the Gauss representation.}

Lemma 4.4. The Gauss representation of critical elasticas is continuous at each point.

Remark 4.5. If $\alpha$ is not differentiable, we can understand $\dot{\alpha}$ in the generalized way, as the difference between the corresponding upper and lower bounds. Similarly, we can consider the function $\alpha$ as the $L^{2}$-limit of smooth functions and calculate the derivative $\dot{\alpha}$ as the $L^{2}$-limit of the derivatives of these functions.

Proof. If the assertion of Lemma 4.4 is not true, then there exists a constant $C$ such that for any $\varepsilon>0$ there is a $t_{0}$ such that

$$
\int_{t_{0}}^{t_{0}+\varepsilon}|\dot{\alpha}| d t>C
$$

(the integral in the left-hand side of the inequality can be infinite). Hence

$$
\int_{t_{0}}^{t_{0}+\varepsilon} \dot{\alpha}^{2} d t>\frac{C^{2}}{\varepsilon}
$$

Therefore, the Euler functional is infinite for this curve. We come to a contradiction.

4.2.2. Variations $\hat{\beta}_{a, \varepsilon, b, \xi}$. Denote by $\delta(x)$ the generalized Dirac $\delta$-function. By definition, let us put

$$
\hat{\beta}_{a, \varepsilon, b, \xi}(x)=\frac{1}{b-a} \int_{0}^{x} \int_{0}^{y} \frac{\delta(z-b-\xi)-\delta(z-b)}{\xi}-\frac{\delta(z-a-\varepsilon)-\delta(z-a)}{\varepsilon} d z d y
$$

In addition, we extend this function as follows

$$
\begin{aligned}
& \hat{\beta}_{a, 0, b, \xi}(x)=\frac{1}{b-a} \int_{0}^{x}\left(\int_{0}^{y} \frac{\delta(z-b-\xi)-\delta(z-b)}{\xi} d z-\delta(y-a)\right) d y \\
& \hat{\beta}_{a, 0, b, 0}(x)=\frac{1}{b-a} \int_{0}^{x}(\delta(y-b)-\delta(y-a)) d y
\end{aligned}
$$

Let

$$
\beta_{a, \varepsilon, b, \xi}=\hat{\beta}_{a, \varepsilon, b, \xi}-h_{\alpha}\left(\hat{\beta}_{a, \varepsilon, b, \xi}\right),
$$

where $h_{\alpha}$ is the orthogonal projection from the space of $L^{2}$-functions to the vector space spanned by the functions $\cos (\alpha(x)), \sin (\alpha(x))$ and constant functions.

Lemma 4.6. The function $\beta_{a, \varepsilon, b, \xi}$ is continuous in the $L^{2}$-norm at all points in which we have already defined it.

The proof is straightforward, so we omit it.

Finally, we put $\hat{\beta}_{a, 0, a, 0}(x)=\delta(x-a)$. 
4.2.3. Orthogonal basis in $H_{\alpha}$. Denote by $H_{\alpha}$ the linear span of the functions $\cos \alpha$, $\sin \alpha$ and constant functions. For an arbitrary choice of $\alpha$, let us fix an orthogonal basis:

$$
\begin{aligned}
e_{\alpha, 1}= & \cos \alpha ; \\
e_{\alpha, 2}= & \sin \alpha+\frac{\int_{0}^{2 \pi} \sin \alpha(x) \cos \alpha(x) d x}{\int_{0}^{2 \pi} \sin ^{2} \alpha(x) d x} \cos \alpha ; \\
e_{\alpha, 3}= & 1+\frac{\int_{0}^{2 \pi} \sin \alpha(x) \cos \alpha(x) d x \int_{0}^{2 \pi} \cos \alpha(x) d x-\int_{0}^{2 \pi} \cos ^{2} \alpha(x) d x \int_{0}^{2 \pi} \sin \alpha(x) d x}{\int_{0}^{2 \pi} \sin ^{2} \alpha(x) d x \int_{0}^{2 \pi} \cos ^{2} \alpha(x) d x-\left(\int_{0}^{2 \pi} \sin \alpha(x) \cos \alpha(x) d x\right)^{2}} \sin \alpha+ \\
& \frac{\int_{0}^{2 \pi} \sin \alpha(x) \cos \alpha(x) d x \int_{0}^{2 \pi} \sin \alpha(x) d x-\int_{0}^{2 \pi} \sin ^{2} \alpha(x) d x \int_{0}^{2 \pi} \cos \alpha(x) d x}{\int_{0}^{2 \pi} \sin ^{2} \alpha(x) d x \int_{0}^{2 \pi} \cos ^{2} \alpha(x) d x-\left(\int_{0}^{2 \pi} \sin \alpha(x) \cos \alpha(x) d x\right)^{2}} \cos \alpha .
\end{aligned}
$$

The Gauss representation for closed curves is not constant, therefore the denominator of the second coefficient for $e_{\alpha, 2}$ is nonzero. By the Cauchy-Schwarz inequality for $L^{2}$-functions, the denominators of the coefficients of $e_{\alpha, 3}$ are also nonzero. The following statement is straightforward.

Lemma 4.7. The functions $e_{\alpha, 1}, e_{\alpha, 2}$, and $e_{\alpha, 3}$ are smooth bounded nonzero functions.

As a corollary we have the following.

Corollary 4.8. The function $h_{\alpha}\left(\hat{\beta}_{t, \varepsilon, u, \xi}\right)(x)$ is a continuous function that $L^{2}$-continuously depends on the parameters $(t, \varepsilon, u, \xi)$ for an arbitrary 4-tuple of parameters satisfying $0 \leq t, u \leq 2 \pi$ (including $(t, 0, t, 0))$.

\subsubsection{Continuous differentiability of the Gauss representation.}

Lemma 4.9. The Gauss representation of a critical elastica is continuously differentiable at each point.

Proof. Let us fix $t \neq u$, and $\xi$, and vary $\varepsilon$. From Equation 4 it follows that

$$
\frac{\alpha(t+\varepsilon)-\alpha(t)}{\varepsilon}=\frac{\alpha(u+\xi)-\alpha(u)}{\xi}+(t-u) \int_{0}^{2 \pi} \alpha(x)\left(h_{\alpha}\left(\hat{\beta}_{t, \varepsilon, u, \xi}\right)(x)\right)^{\prime \prime} d x .
$$

The first summand of the left part does not depend on $\varepsilon$. The second summand is a continuous function in the $\varepsilon$ variable, since $\alpha$ is continuous. Therefore, the limit of the expression in the right-hand side of the equality exists, i.e., the derivative $\dot{\alpha}$ exists at $t$.

Let us fix $t$, and vary $\varepsilon$. From Equation 4 it follows that

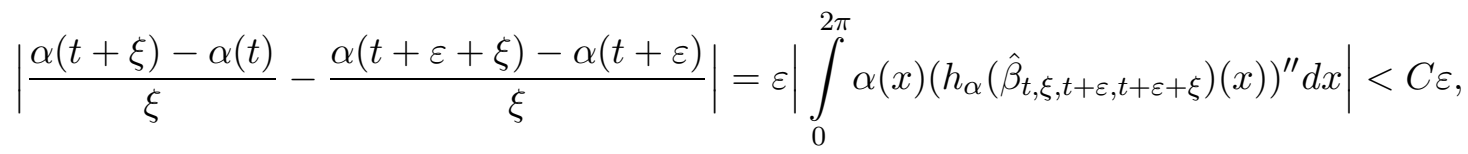

where $C$ does not depend on $\varepsilon$ and $\xi$. Therefore,

$$
|\dot{\alpha}(t)-\dot{\alpha}(t+\varepsilon)|<C \varepsilon .
$$

Thus, the function $\dot{\alpha}$ is continuous at $t$. 
4.2.5. Conclusion of the proof of Proposition 4.3. From Proposition 4.9 it follows that the Gauss representation of a critical elastica is continuously differentiable at each point.

From Equation 3 it follows that

$$
\frac{\dot{\alpha}(t+\varepsilon)-\dot{\alpha}(t)}{\varepsilon}=\int_{0}^{2 \pi} \dot{\alpha}(x)\left(h_{\alpha}\left(\hat{\beta}_{t, 0, t+\varepsilon, 0}\right)(x)\right)^{\prime} d x .
$$

Therefore,

$$
\ddot{\alpha}(t)=\lim _{\varepsilon \rightarrow 0} \int_{0}^{2 \pi} \dot{\alpha}(x)\left(h_{\alpha}\left(\hat{\beta}_{t, 0, t+\varepsilon, 0}\right)(x)\right)^{\prime} d x .
$$

Corollary 4.8 implies that the function $h_{\alpha}\left(\hat{\beta}_{t, \varepsilon, u, \xi}\right)(x) L^{2}$-continuously depends on $\varepsilon$. Therefore, the limit exists. Hence the Gauss representation is twice differentiable.

4.3. Uniqueness of $\infty$-shaped normal forms passed once. In this section we briefly analyze the critical closed elasticae whose Gauss representations satisfy the equation

$$
\ddot{\alpha}+\sin \alpha=0 .
$$

It is clear that the elasticae whose Gauss representations correspond to the motions of the pendulum that makes complete turns are not bounded. If the pendulum does not make a complete turn, then it is possible to get a closed elastica. All such curves are homotopic to the figure " $\infty$ " curve. We call them $\infty$-shaped normal forms. The main statement about $\infty$-shaped normal forms is as follows.

Proposition 4.10. All $\infty$-shaped normal forms are homothetic to each other.

In the proof of Proposition 4.10, we essentially used the following general statement.

Proposition 4.11. (On 2-germ similarity.) Consider two $\mathcal{C}^{2}$-curves $\gamma_{1}:\left[0, T_{1}\right] \rightarrow \mathbb{R}^{2}$ and $\gamma_{2}:\left[0, T_{2}\right] \rightarrow \mathbb{R}^{2}$ with Gauss representations $\alpha_{1}$ and $\alpha_{2}$ respectively.

Suppose that the following conditions hold:

- the curves are convex;

- the curves are inscribed in the same angle centered at $O$;

- the curves have the same starting point: $\gamma_{1}(0)=\gamma_{2}(0)$.

Then there exists a pair of points $\left(t_{1}, t_{2}\right)$ simultaneously satisfying

$$
\alpha_{1}\left(t_{1}\right)=\alpha_{2}\left(t_{2}\right) \quad \text { and } \quad \dot{\alpha}_{1}\left(t_{1}\right)=\dot{\alpha}_{2}\left(t_{2}\right) .
$$

We start with the following two lemmas.

Lemma 4.12. Consider two $\mathcal{C}^{2}$-curves $\gamma_{1}:\left[0, T_{1}\right] \rightarrow \mathbb{R}^{2}$ and $\gamma_{2}:\left[0, T_{2}\right] \rightarrow \mathbb{R}^{2}$ with Gauss representations $\alpha_{1}$ and $\alpha_{2}$ respectively.

Suppose that the following conditions hold:

- the curves are convex;

- the curves are inscribed in the same angle centered at $O$;

- the curves have the same starting point: $\gamma_{1}(0)=\gamma_{2}(0)$.

- for any pair of points $\left(t_{1}, t_{2}\right)$ satisfying $\alpha_{1}\left(t_{1}\right)=\alpha_{2}\left(t_{2}\right)$ the inequality $\dot{\alpha}_{1}\left(t_{1}\right)>\dot{\alpha}_{2}\left(t_{2}\right)$ holds.

Then the point $\gamma_{1}\left(T_{1}\right)$ is contained in the interior of the segment with endpoints $O$ and $\gamma_{2}\left(T_{2}\right)$ (see Figure 1, left). 

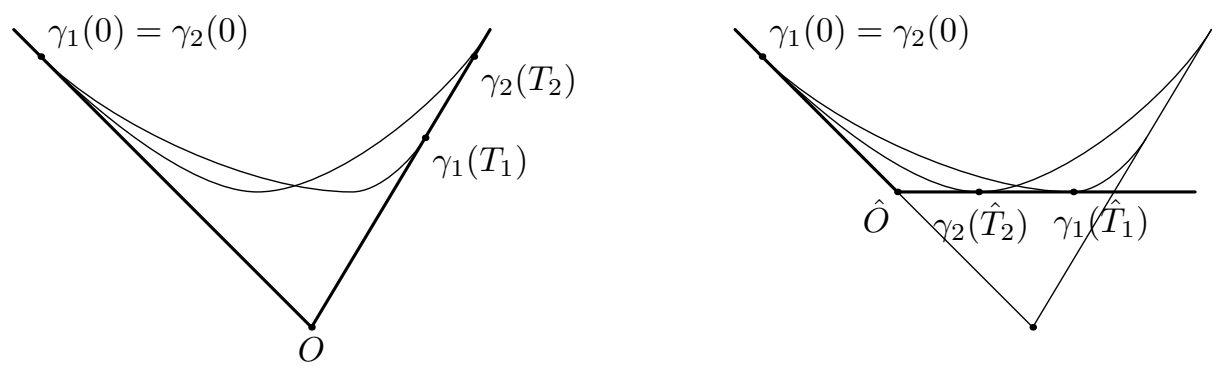

Figure 1. Two curves inscribed at the same angle (left) and their common tangent line (right).

Proof. Without loss of generality, we assume that the angle in which both curves are inscribed is defined by the rays $y=\tan \alpha_{0}, x>0$ and $y=-\tan \alpha_{0}, x<0$. Let the starting point be on the left ray. So both $\alpha_{1}$ and $\alpha_{2}$ are increasing functions from $-\alpha_{0}$ to $\alpha_{0}$.

The condition that for any pair of points $\left(t_{1}, t_{2}\right)$ satisfying $\alpha_{1}\left(t_{1}\right)=\alpha_{2}\left(t_{2}\right)$ the inequality $\dot{\alpha}_{1}\left(t_{1}\right)>\dot{\alpha}_{2}\left(t_{2}\right)$ holds implies that

$$
\int_{0}^{T_{1}} \cos \alpha_{1}(t) d t<\int_{0}^{T_{2}} \cos \alpha_{2}(t) d t .
$$

Hence the $x$-coordinate of $\alpha_{1}\left(T_{1}\right)$ is smaller than the $x$-coordinate of $\alpha_{2}\left(T_{2}\right)$. Therefore, the point $\gamma_{1}\left(T_{1}\right)$ is contained in the interior of the segment with endpoints $O$ and $\gamma_{2}\left(T_{2}\right)$.

Lemma 4.13. There are no $C^{2}$-curves satisfying all the conditions of Lemma 4.12.

Proof. We prove this by reductio ad absurdum. Suppose that such curves $\gamma_{1}$ and $\gamma_{2}$ exist.

On the one hand, by Lemma 4.12 the point $\gamma_{1}\left(T_{1}\right)$ is contained in the interior of the segment with endpoints $O$ and $\gamma_{2}\left(T_{2}\right)$. On the other hand, the condition $\dot{\alpha}_{1}(0)>\dot{\alpha}_{2}(0)$ implies that there are some points $\gamma_{2}(t)$ (with small parameter $t$ ) that lie in the same connected component in the complement of the angle to the curve $\gamma_{1}$. Hence, there is a point where $\gamma_{1}$ crosses $\gamma_{2}$.

Hence there exists a line $l$ which is tangent to both curves $\gamma_{1}$ and $\gamma_{2}$. Suppose that this happens at times $\hat{T}_{1}$ and $\hat{T}_{2}$ respectively. Let $l$ cross the left ray $r$ at the point $\hat{O}$ (see Figure 1 , right). Notice that in these settings, the point $\gamma_{2}\left(\hat{T}_{2}\right)$ is contained in the interior of the segment with endpoints $\hat{O}$ and $\gamma_{1}\left(\hat{T}_{1}\right)$. We come to a contradiction with Lemma 4.12 for the curves $\gamma_{1}:\left[0, \hat{T}_{1}\right] \rightarrow \mathbb{R}^{2}$ and $\gamma_{2}:\left[0, \hat{T}_{2}\right] \rightarrow \mathbb{R}^{2}$, which are both inscribed in the angle with vertex $\hat{O}$.

Proof of Proposition 4.11. From Lemma 4.13, it follows that if all the conditions are satisfied, then there are two pairs $\left(t_{1}, t_{2}\right)$ and $\left(t_{3}, t_{4}\right)$ such that

$$
\left\{\begin{array} { l } 
{ \alpha _ { 1 } ( t _ { 1 } ) = \alpha _ { 2 } ( t _ { 2 } ) } \\
{ \dot { \alpha } _ { 1 } ( t _ { 1 } ) > \dot { \alpha } _ { 2 } ( t _ { 2 } ) }
\end{array} \quad \text { and } \quad \left\{\begin{array}{l}
\alpha_{1}\left(t_{3}\right)=\alpha_{2}\left(t_{4}\right) \\
\dot{\alpha}_{1}\left(t_{3}\right)<\dot{\alpha}_{2}\left(t_{4}\right)
\end{array}\right.\right. \text {. }
$$

Hence, for continuity reasons, there exists a pair $\left(t_{5}, t_{6}\right)$ for which we have both

$$
\alpha_{1}\left(t_{1}\right)=\alpha_{2}\left(t_{2}\right) \quad \text { and } \quad \dot{\alpha}_{1}\left(t_{5}\right)=\dot{\alpha}_{2}\left(t_{6}\right) .
$$

Lemma 4.14. The duration of the period increases with the amplitude. 

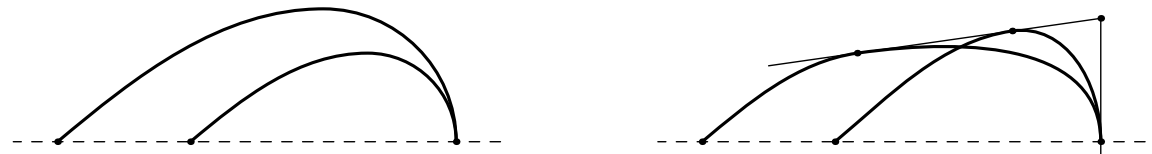

Figure 2. The curves $\gamma_{1}$ and $\gamma_{2}$ either do not intersect (on the left) or intersect (on the right). In the last case some of their parts are inscribed in the same angle.

Proof. This follows directly from the fact that the period $T$ with amplitude $\alpha_{0}$ is calculated by the formula:

$$
T=4 \omega K\left(\sin ^{2} \frac{\alpha_{0}}{2}\right)=4 \omega \int_{0}^{\pi / 2} \frac{d \theta}{\sqrt{1-\sin ^{2} \frac{\alpha_{0}}{2} \sin ^{2} \theta}}
$$

(here $K(t)$ is the complete elliptic integral of the first type). The function under the integral sign increases when $\alpha_{0}$ increases.

Proof of Proposition 4.10. We argue by reduction ad absurdum. Suppose that there are two different closed $\infty$-shaped solutions of the equation

$$
\ddot{\alpha}+\sin \alpha=0
$$

with amplitudes $\alpha_{1}$ and $\alpha_{2}$. Let $\alpha_{2}>\alpha_{1}$. Consider the two curves $\gamma_{1}$ and $\gamma_{2}$ corresponding to one fourth of the $\infty$-shaped curves, starting from the point with vertical tangent vector (see Figure 2).

Let us prove that $\gamma_{1}$ and $\gamma_{2}$ do not intersect at interior points. Suppose that the converse is true. If the curves $\gamma_{1}$ and $\gamma_{2}$ intersect, then there is some line which is tangent to both of these curves. Therefore, there are some parts of them that are inscribed in the same angle (see Figure 2, right). On the one hand, by Proposition 4.11 there exists a pair of points $\left(t_{1}, t_{2}\right)$ satisfying simultaneously

$$
\alpha_{1}\left(t_{1}\right)=\alpha_{2}\left(t_{2}\right) \quad \text { and } \quad \dot{\alpha}_{1}=\dot{\alpha}_{1}\left(t_{1}\right)=\dot{\alpha}_{2}\left(t_{2}\right) .
$$

On the other hand, any solution of the pendulum equation $(\ddot{\alpha}+\sin \alpha=0)$ satisfies

$$
\dot{\alpha}= \pm \sqrt{\cos \alpha+C} .
$$

Combining these, we obtain the following:

$$
\sqrt{\cos \alpha_{2}\left(t_{1}\right)+C_{1}}=\sqrt{\cos \alpha_{1}\left(t_{1}\right)+C_{1}}=\dot{\alpha}_{1}\left(t_{1}\right)=\dot{\alpha}_{2}\left(t_{2}\right)=\sqrt{\cos \alpha_{2}\left(t_{1}\right)+C_{2}},
$$

which implies $C_{1}=C_{2}$. Hence the curves $\gamma_{1}$ and $\gamma_{2}$ coincide. We come to a contradiction. Therefore, the curves $\gamma_{1}$ and $\gamma_{2}$ do not intersect at inner points.

Since the curves do not intersect and $\dot{\alpha}_{1}(0)<\dot{\alpha}_{2}(0)$, the curve $\gamma_{1}$ lies above the curve $\gamma_{2}$. On the one hand, since $\gamma_{1}$ is convex, and both ends of both curves are on the $O X$ coordinate axis, the length of $\gamma_{1}$ is not less than the length of $\gamma_{2}$. On the other hand, Lemma 4.14 implies that the length of $\gamma_{1}$ is less than the length of $\gamma_{2}$. We come to a contradiction. 
Remark 4.15. The unique $\infty$-shaped curve is called the Bernoulli closed elastica. Its arc length representation is closely related to Bernoulli's lemniscate. In [12] and [21] there are good historical overviews about elasticae in general, which contain, in particular, good descriptions of this curve.

4.4. Unstable critical elasticae for the Euler functional. In this subsection, we prove the following statement.

Proposition 4.16. Bernoulli's closed elastica passed several times is not stable.

Construction of $\Gamma_{\varepsilon}$. Consider the loop of the closed elastica with center $O_{1}$ at the origin with nonpositive first coordinate. Construct another loop of Bernoulli's closed elastica centrally symmetric to the first one and tangent to the first loop at the point $A$ with first coordinate $-\varepsilon / 2$ and positive second coordinate (see Figure 3, left). Denote the center of the second loop by $\mathrm{O}_{2}$. Find the point $\mathrm{O}_{3}$ on the $\mathrm{OY}$-axis such that the line $\mathrm{O}_{2} \mathrm{O}_{3}$ touches the upper branch of the elastica at $\mathrm{O}_{2}$ and connect $\mathrm{O}_{3}$ with $\mathrm{O}_{2}$ by a line segment (Figure 3, right).

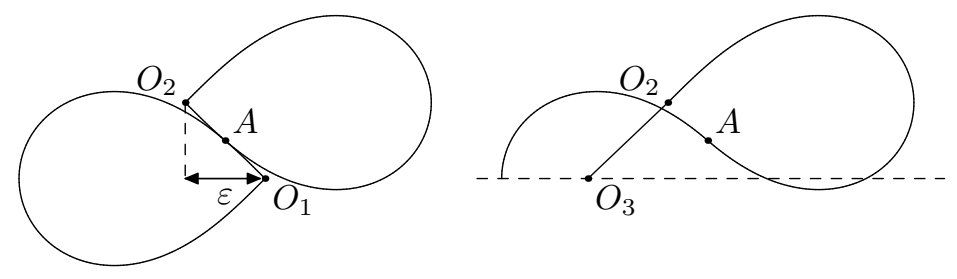

Figure 3. Preliminary steps to construct $\Gamma_{\varepsilon}$.

Finally, add the symmetric picture about the $O Y$-axis and add one more Bernoulli closed elastica loop centered at $O_{3}$, as on Figure 4, left. Denote the symmetric point to $\mathrm{O}_{2}$ by $\mathrm{O}_{4}$.
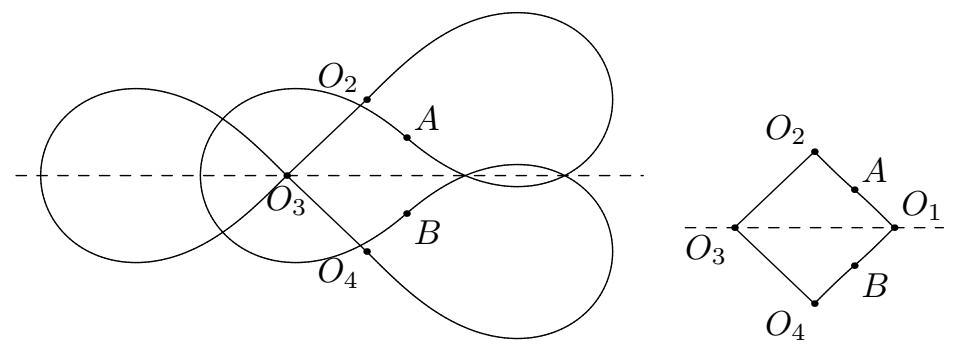

Figure 4. The embedding $\Gamma_{\varepsilon}$ and its difference from the union of four Bernoulli's closed elastica loops.

Remark. The immersion $\Gamma_{\varepsilon}$ is not $C^{\infty}$-smooth but only $C^{1}$-smooth. We omit standard smoothing procedures here.

Proof of Proposition 4.16. Let us briefly estimate the difference in lengths and curvatures between $\Gamma_{\varepsilon}$ and the double Bernoulli closed elastica (i.e. the union of the four loops of Bernoulli's closed elastica). 
First, we compare the lengths. If we replace two segments $\mathrm{O}_{2} \mathrm{O}_{3}$ and $\mathrm{O}_{3} \mathrm{O}_{4}$ in $\Gamma_{\varepsilon}$ by the curves $\mathrm{O}_{1} \mathrm{AO}_{2}$ and $\mathrm{O}_{3} \mathrm{BO}_{4}$ constructed via the corresponding parts of Bernoulli's closed elastica (Figure 4, right), then the length will be exactly equal to twice the length of the closed elastica. Notice that the angle $\mathrm{O}_{2} \mathrm{O}_{1} \mathrm{O}_{3}$ is less than the angle $\mathrm{O}_{2} \mathrm{O}_{3} \mathrm{O}_{1}$, since all the absolute values of the derivatives at points of the closed elastica are greater than $\tan \left(\mathrm{O}_{2} \mathrm{O}_{3} \mathrm{O}_{1}\right)$ almost everywhere. Thus the length of $\mathrm{O}_{1} \mathrm{O}_{2}$ is greater than the length of $\mathrm{O}_{3} \mathrm{O}_{2}$. Hence the length of $\mathrm{O}_{3} \mathrm{O}_{2}$ is less than the length of the curve $O_{1} A O_{2}$. For the same reason, the length of $O_{3} O_{4}$ is less than the length of the curve $\mathrm{O}_{1} \mathrm{BO}_{4}$. Therefore, the length of $\Gamma_{\varepsilon}$ is smaller than the length of the double Bernoulli closed elastica. The curvature of the Bernoulli closed elastica part of $\Gamma_{\varepsilon}$ (the bold $\infty$-curve in Figure 5) coincides with the curvatures of the corresponding points on the double Bernoulli's closed elastica. The curvature at points on the additional segments are equal to zero. Since the lengths are smaller and the curvatures at the corresponding points are not greater, for the total Euler functional we have

$$
U\left(\Gamma_{\varepsilon}\right)<U\left(\Gamma_{0}\right)
$$

Hence, the double Bernoulli's closed elastica is a saddle point of the configuration space of all immersions of 0 index.

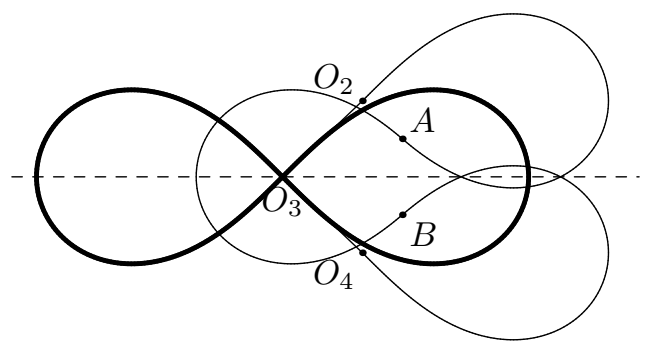

Figure 5. The immersion $\Gamma_{\varepsilon}$ for n-tuple Bernoulli's closed elasticae.

In the case of an $n$-tuple Bernoulli closed elastica for $n>2$, we add $(n-2)$-tuple Bernoulli closed elasticae to all the immersions of the above construction of the energy reducing deformation for the double Bernoulli closed elastica, as it is shown on Figure 5. The $(n-2)$-tuple Bernoulli closed elastica remains unchanged during the whole deformation.

4.5. Proof of Theorem 1.3 and Theorem 2.2. In this subsection, we conclude the proofs of the main theorems.

4.5.1. Proof of Theorem 1.3. (i) By Proposition 4.3, the Gauss representation of any critical elastica is twice differentiable at any point. By Corollary 4.1 (see also Remark 4.2) all critical elasticas with twice differentiable Gauss representation are either circles or satisfy the equation of the simple pendulum. By Proposition 4.10, all critical elasticae whose Gauss representation satisfy the equation of the simple pendulum are homothetic to Bernoulli's closed elastica (see Remark 4.15). The first item is proved.

(ii) We have proved this item in Proposition 4.16.

(iii) The first two items of this theorem imply that each connected component has a unique stable critical point. Hence these unique critical points are local minima. Recall that in the case of a nonzero Whitney index, the corresponding curve is a circle passed a number of times equal 
to the Whitney index (the orientation is determined by the sign of the index). If the Whitney index is zero, then the critical elastica is the Bernoulli closed $\infty$-shaped elastica. This concludes the proof of Theorem 1.3.

4.5.2. Proof of Theorem 2.2. Theorem 1.3 implies that each regular homotopy class of regular curves contains a unique normal form.

Acknowledgements. We are grateful to Mikhail Zelikin for an illuminating informal talk about Euler elasticae, to Alexander Demidov for pointing out the references [18] and [19], and to Robert Goldstein for a useful discussion of the present paper. Oleg Karpenkov is supported by the Austrian Science Fund (FWF), grant M 1273-N18. Alexey Sossinsky is partially supported by the RFBR-CNRS-a grant \#10-01-93111 and the RFBR grant \# 12-01-00748-a.

\section{REFERENCES}

[1] S. Avvakumov, software available at http://www.mccme.ru/knotenergy

[2] S. Bryson, M. H. Freedman, Z.-X. He, and Z. Wang, Möbius invariance of knot energy, Bull. Amer. Math. Soc. (N.S.), vol. 28 (1993), no. 1, pp. 99-103.

[3] L. Euler, Methodus inveniendi lineas cyrvas maximi minimive proprietate gaudentes, sive Solutio problematis isoperimitrici latissimo sensu accepti, Lausanne, Genève, 1744.

[4] M. H. Freedman, Z.-X. He, Links of tori and the energy of incompressible flows, Topology vol. 30 (1991), no. 2, pp. 283-287.

[5] M. H. Freedman, Z.-X. He, and Z. Wang, Möbius energy of knots and unknots, Ann. of Math. (2), vol. 139 (1994), no. 1, pp. 1-50.

[6] W. Fukuhara, Energy of a knot, The fête of topology, Academic Press, (1988), pp 443-451.

[7] O. Karpenkov, A. Sossinsky, Energies of knot diagrams, Russian J. of Math. Phys., vol. 18(2011), no. 3 , pp. 306-317.

[8] O. Karpenkov, Energy of a knot: variational principles, Russian J. of Math. Phys. vol. 9(2002), no. 3, pp. $275-287$.

[9] O. Karpenkov, Energy of a knot: some new aspects Fundamental Mathematics Today, Nezavis. Mosk. Univ., Moscow (2003), pp. 214-223.

[10] O. Karpenkov, The Möbius energy of graphs, Math. Notes, vol. 79(2006), no. 1-2, pp. 134-138.

[11] D. Kim, R. Kusner, Torus Knots Extremizing the Möbius Energy, Experimental Mathematics, vol. 2(1993), no. 1, pp. 1-9.

[12] R. Levien, The elastica: a mathematical history, EECS Department, University of California, Berkeley, Technical Report No. UCB/EECS-2008-103, 2008. http://www.eecs.berkeley.edu/Pubs/TechRpts/2008/EECS-2008-103.html

[13] H. K. Moffat, The degree of knottedness of tangled vortex lines, J. Fluid Mech. vol. 35(1969), pp. 117129.

[14] J. O'Hara, Energy of a knot, Topology, vol. 30(1991), no. 2, pp. 241-247.

[15] J. O'Hara, Family of energy functionals of knots, Topology Appl. vol 48(1992), no. 2, pp. 147-161.

[16] J. O'Hara, Energy functionals of knots II, Topology Appl. vol. 56(1994), no. 1, pp. 45-61.

[17] J. O'Hara, Energy of Knots and Conformal Geometry, K \& E Series on Knots and Everything Vol. 33, World Scientific, 2003, 288 p.

[18] Yu.S.Osipov, M.I.Zelikin, Higher-order Euler elastics and elastic hulls, Russ.J. Math.Phys. 19, no. 2, 163-172 (2012)

[19] Yu.L.Sachkov, Closed Euler elasticae, Progam Syst.Inst. Preprint, 2011.

[20] A. B. Sossinsky, Mechanical Normal Forms of Knots and Flat Knots, Russ. J. Math. Phys. vol. 18, no. 2(2011).

[21] R. Sridharan, Physics to mathematics: from lintearia to lemniscate - I, Resonance(2004), pp. 21-29. 
E-mail address, Oleg Karpenkov: karpenkov@tugraz.at

E-mail address, Alexey Sossinsky: asossinsky@yandex.ru

E-mail address, Sergey Avvakumov: s.avvakumov@gmail.com 